\title{
New Insight into the Isomorphism of Polynomial Problem IP1S and Its Use in Cryptography
}

\author{
Gilles Macario-Rat ${ }^{1}$, Jérôme Plut ${ }^{2}$, and Henri Gilbert ${ }^{2}$ \\ 1 Orange Labs \\ 38-40, rue du Général Leclerc, 92794 Issy-les-Moulineaux Cedex 9, France \\ gilles.macariorat@orange.com \\ 2 ANSSI, \\ 51 Boulevard de la Tour-Maubourg, 75007 Paris, France \\ \{henri.gilbert, jerome.plut\}@ssi.gouv.fr
}

\begin{abstract}
This paper investigates the mathematical structure of the "Isomorphism of Polynomial with One Secret" problem (IP1S). Our purpose is to understand why for practical parameter values of IP1S most random instances are easily solvable (as first observed by Bouillaguet et al.). We show that the structure of the equations is directly linked to a matrix derived from the polar form of the polynomials. We prove that in the likely case where this matrix is cyclic, the problem can be solved in polynomial time - using an algorithm that unlike previous solving techniques is not based upon Gröbner basis computation.
\end{abstract}

\section{Introduction}

Multivariate cryptography is a sub area of cryptography the development of which was initiated in the late 80's [13] and was motivated by the search for alternatives to asymmetric cryptosystems based on algebraic number theory. RSA and more generally most existing asymmetric schemes based on algebraic number theory use the difficulty of solving one univariate equation over a large group (e.g. $x^{e}=y$ where $e$ and $y$ are known). Multivariate cryptography as for it, aims at using the difficulty of solving systems of multivariate equations over a small field.

A limited number of multivariate problems have emerged that can be reasonably conjectured to possess intractable instances of relatively small size. Two classes of multivariate problems are underlying most multivariate cryptosystems proposed so far, the MQ problem of solving a multivariate system of $m$ quadratic equations in $n$ variables over a finite field $\mathbb{F}_{q}$ - that was shown to be NP-complete even over $\mathbb{F}_{2}$ for $m \approx n[10$ - and the broad family of the so-called isomorphism of polynomials (IP) problems.

Isomorphism of Polynomial problems can be roughly described as the equivalence of multivariate polynomial systems of equations up to linear (or affine) bijective changes of variables. Two separate subfamilies of IP problems can be distinguished: isomorphism of polynomials with two secrets (IP2S for short) and isomorphism of polynomials with one secret (IP1S for short). A little more in 
detail, given two $m$-tuples $a=\left(a_{1}, \ldots, a_{m}\right)$ and $b=\left(b_{1}, \ldots, b_{m}\right)$ of polynomials in $n$ variables over $\mathbb{K}=\mathbb{F}_{q}$, IP2S consists of finding two linear bijective transformations $S$ of $\mathbb{K}^{n}$ and $T$ of $\mathbb{K}^{m}$, such that $b=T \circ a \circ S$. Respectively, (computational) IP1S consists of finding one linear bijective transformations $S$ of $\mathbb{K}^{n}$, such that $b=a \circ S$. Many variants of both problems can be defined depending on the value of the triplet $(n, m, q)$, the degree $d$ of the polynomial equations of $a$ and $b$, whether these polynomials are homogeneous or not, whether $S$ and $T$ are affine or linear, etc. It turns out that there are considerable security and simplicity advantages in restricting oneself, for cryptographic applications, to instances involving only homogeneous polynomials of degree $d$ and linear transformations $S$ and $T$. For performance reasons, the quadratic case $d=2$ is most frequently encountered in cryptography. Due to the existence of an efficient canonical reduction algorithm for quadratic forms, instances such that $m \geq 2$ must then be considered. The cubic case $d=3$ is also sometimes considered, then instances such that $m=1$ are generally encountered.

Many asymmetric cryptosystems whose security is related to the hardness of special trapdoor instances of IP2S were proposed in which all or part of the $m$-tuple of polynomials $b$ plays the role of the public key and is related by secret linear bijections $S$ and $T$ to a specially crafted, easy to invert multivariate polynomial mapping $a$. Most of these systems, e.g. Matsumoto and Imai's seminal multivariate scheme $\mathrm{C}^{*}$ 13, but also reinforced variants such as SFLASH and HFE [18]16] were shown to be weak because the use of trapdoor instances of IP2S with specific algebraic properties considerably weakens the general IP2S problem. A survey of the status of the IP2S problems and improved techniques for solving homogeneous instances are presented in [1] and [4.

The IP1S problem was introduced in [16] by Patarin, who proposed in the same paper a zero-knowledge asymmetric authentication scheme named the IP identification scheme with one secret (IP1S scheme for short). This authentication scheme is inspired by the well known zero-knowledge proof for Graph Isomorphism by Goldreich et al. [11. It can be converted into a (less practical) asymmetric signature scheme using the Fiat-Shamir transformation. The IP1S problem and the related identification scheme were believed to possess several attractive features:

- The conjecture that the IP1S problem is not solvable in polynomial time was supported by the proof in [17] that the quadratic version of IP1S (QIP1S for short) is at least as hard as the Graph Isomorphism problem (GI) 1 1 , one of the most extensively studied problems in complexity theory. While the GI problem is not believed to be NP-complete since it is NP and co-NP and hard instances of GI are difficult to construct for small parameter values, GI is generally believed not to be solvable in polynomial time.

- unlike the encryption or signature schemes based on IP2S mentioned above, the IP1S scheme does not use special trapdoor instances of the IP1S problem

${ }^{1}$ However as mentioned in the conclusion of this paper, if the flaw recently discovered by the authors in the corresponding proof in [17] is confirmed, this casts some doubts on the fact that Quadratic IP1S is indeed as hard as GI. 
and therefore its security is directly related to the intractability of general IP1S instances.

The IP1S problem also has some loose connections with the multivariate signature scheme UOV 12 , that has until now remarkably well survived all advances in the cryptanalysis of multivariate schemes. While in UOV the public quadratic function $b$ is related to the secret quadratic function by the equation $b=a \circ S$, both $a$ and $S$ are unknown whereas only $S$ is unknown in the IP1S problem.

Former Results. Initial assessments of the security of practical instances of the IP1S problem suggested that relatively small public key and secret sizes typically about 256 bits - could suffice to ensure a security level of more than $2^{64}$. The IP1S scheme therefore appeared to favorably compare with many other zeroknowledge authentication schemes, e.g [2122 20]. Moreover, despite advances in solving some particular instances of the IP1S problem, in particular Perret's Jacobian algorithm 2 [19], the four challenge parameter values proposed in 1996 [16] (with $q=2$ or $2^{16}, d=2$ and $m=2$, or $d=3$ and $m=1$ ) remained unbroken until 2011.

Significant advances on solving IP1S instances that are practically relevant for cryptography were made quite recently [21]. Dubois in [7] and the authors of [2] were the first to notice that the IP1S problem induces numerous linear equations in the coefficients of the matrix of $\mathrm{S}$ and of the inverse mapping $T=S^{-1}$. When $m \geq 3$, the number $m n^{2}$ of obtained linear equations is substantially larger than the number $2 n^{2}$ of variables. While the system cannot have full rank since the dimension of the vector space of solutions is at least 1 , it can heuristically be expected to have a very small vector space of solutions that can be tried exhaustively. The authors of [2] even state that they "empirically find one solution (when the polynomials are randomly chosen)".

Therefore the most interesting remaining case appears to be $m=2$. It is shown in [2] that the vector space of solutions of the linear equations is then isomorphic to the commutant of a non-singular $n \times n$ matrix $M$ and that its dimension $r$ is lower bounded by $n$ in odd characteristic and $2 n$ in even characteristic. The reported computer experiments indicate that $r$ is extremely likely to be close to these lower bounds in practice. While for typical values of $q^{n}$ the vector space of solutions is too large to be exhaustively searched, one can try to solve the equation $b=a \circ S$ over this vector space. This provides a system of quadratic equations in a restricted variable set of $r \approx n$ (resp. $r \approx 2 n$ ) coordinates. The approach followed in 2 in order to solve this system consisted of applying Gröbner basis algorithms such as Faugère's F4 8] and related computer algebra tools such as FGLM 9. This method turned out to be quite successful: all the IP1S challenges proposed by Patarin were eventually broken in computing times ranging from less than $1 \mathrm{~s}$ to 1 month. This led the authors of [2] to conclude that "[the] IP1S-Based identification scheme is no longer competitive

${ }^{2}$ This algorithm recovers $m n$ linear equations in the coefficients of $S$ and is therefore suited for solving IP1S instances such that $m \approx n$. 
with respect to other combinatorial-based identification schemes". However, the heuristic explanation suggested in [2, namely that the obtained system was so massively over defined that a random system with the same number of random quadratic equations would be efficiently solvable in time $O\left(n^{9}\right)$ with overwhelming probability, was later on shown to be false by one of the authors of [2, due to an overestimate of the number of linearly independent quadratic equations.

This is addressed in Bouillaguet's PhD dissertation [1] where the results of 2] are revisited. The main discrepancy with the findings of [2] is the observation that in all the reported experiments in odd and even characteristic, the number of linearly independent quadratic equations, that was supposed in [2] to be close to $n^{2}$, is actually bounded over by a small multiple of $n$ and only marginally larger than $r$. The author writes "This means that we cannot argue that solving these equations is doable in polynomial time. An explanation of this phenomenon has eluded us so far." Despite of the surprisingly small number of linearly independent quadratic equations, nearly all instances are confirmed to be efficiently solvable for all practical values of $n$ when the size $q$ of the field is sufficiently small ( $q=2$ or 3 ) and still solvable efficiently up to values of $n$ of about 20. The author writes "For instance, when $q=2$ and $n=128$ we are solving a system of 256 quadratic equations in 256 variables over $\mathbb{F}_{2}$. When the equations are random this is completely infeasible. In our case, it just takes 3 minutes ! We have no clear explanation of this phenomenon."

Our Contribution. The lack of explanation for the success of the attack - more precisely the puzzling fact that the number of linearly independent quadratic equations is close to $n$ in odd characteristic and to $2 n$ in even characteristic and the even more puzzling fact that nearly all instances are nevertheless solvable motivated our research on IP1S. We revisited the former analysis and eventually found an algebraic explanation of why most random instances of the quadratic IP1S problem are efficiently solvable that leads to a new method (not based on Gröbner basis computations) to directly solve these instances. Our analysis shows in particular that in the likely cases where the characteristic is odd and the matrix $M$ is cyclic or the characteristic is even and $M$ is similar to a blockwise diagonal matrix with two equal cyclic $\frac{n}{2} \times \frac{n}{2}$ diagonal blocks, the quadratic equations split up in an appropriate base in small triangular quadratic systems that can be solved efficiently in polynomial time. The highlighted structure of the quadratic equations seems to be the essential reason why Gröbner basis computations behave so well on most instances.

The rest of this paper is organized as follows. In Section 2, we present the problem IP1S, its background and some major mathematical results used in the following sections. We then discuss in Section 3 and 4 the resolution of the problem over finite fieds of odd, resp. even characteristic. 


\section{The Isomorphism of Polynomial Problem with One Secret}

\subsection{Notations and First Definitions}

Let $\mathbb{K}$ be a field; for practical considerations, we shall assume that $\mathbb{K}$ is the finite field $\mathbb{F}_{q}$ with $q$ elements, although most of the discussion is true in the general case.

A (homogeneous) quadratic form in $n$ variables over $\mathbb{K}$ is a homogeneous polynomial of degree two, of the form $q=\sum_{i, j=1 \ldots n} \alpha_{i, j} x_{i} x_{j}$, where the coefficients $\alpha_{i, j}$ belong to $\mathbb{K}$. For simplicity, we write $x=\left(x_{i}\right)$ for the vector with coordinates $x_{i}$. The quadratic form $q$ can be described by the matrix with general term $\alpha_{i, j}$. Note that the matrix representation of a quadratic form is not unique: two matrices represent the same linear form if, and only if, their difference is skew-symmetric.

The polar form associated to a quadratic form $q$ is the bilinear form $b=\mathcal{P}(q)$ defined by $b(x, y)=q(x+y)-q(x)-q(y)$. This is a symmetric bilinear form. This can be used to give an intrinsic definition of bilinear forms (which is useful to abstract changes of bases from some proofs below): given a vector space $V$, a quadratic form over $V$ is a function $q: V \rightarrow \mathbb{K}$ such that

(i) for all $x \in V$ and $\lambda \in \mathbb{K}, q(\lambda x)=\lambda^{2} q(x)$;

(ii) the polar form $\mathcal{P}(q)$ is bilinear.

For any matrix $A$, let ${ }^{t} A$ be the transpose matrix of $A$ and $\mathcal{P}(A)$ be the symmetric matrix ${ }^{t} A+A$. Then if $q$ is a quadratic form with matrix $A$, its polar form has matrix $\mathcal{P}(A)$. The quadratic form $q$ is regular if its polar form is not singular, i.e. if it defines a bijection from $V$ to its dual. In general, we define the kernel of a quadratic form to be the kernel of its polar form.

From the definition of $b=\mathcal{P}(q)$ we derive the polarity identity

$$
2 q(x)=b(x, x) .
$$

This identity obviously behaves very differently when 2 is a unit in $\mathbb{K}$ and when $2=0$ in $\mathbb{K}$. This forces us to use some quite different methods in both cases.

If 2 is invertible in $\mathbb{K}$ then the polarity identity (11) allows recovery of a quadratic form from its polar bilinear form. In other words, quadratic forms in $n$ variables correspond to symmetric matrices.

Conversely, if $2=0$, then the polarity identity reads as $b(x, x)=0$; in other words, the polar form is an alternating bilinear form. In this case, equality of polar forms does not imply equality of quadratic forms. Define $\Delta(A)$ as the matrix of diagonal entries of the matrix $A$. Then quadratic forms $A$ and $B$ are equal if, and only if, $\mathcal{P}(A)=\mathcal{P}(B)$ and $\Delta(A)=\Delta(B)$.

\subsection{The Quadratic IP1S Problem}

We now state the quadratic IP1S problem and give an account of its current status after the recent work of [2] and [1]. 
Problem 1 (Quadratic IP1S). Given two $m$-tuples $a=\left(a_{1}, \ldots, a_{m}\right)$ and $b=$ $\left(b_{1}, \ldots, b_{m}\right)$ of quadratic homogeneous forms in $n$ variables over $\mathbb{K}=\mathbb{F}_{q}$, find a non-singular linear mapping $S \in G L_{n}(\mathbb{K})$ (if any) such that $b=a \circ S$, i.e. $b_{i}=a_{i} \circ S$ for $i=1, \ldots, m$.

Remark 1. In order not to unnecessarily complicate the presentation, our definition of the IP1S problem slightly differs 3 from the initial statement of the problem introduced in [16]. Though the name "quadratic homogeneous IP1S" might be more accurate to refer to the exact class of instances we consider, we will name it quadratic IP1S or IP1S in the sequel.

If we denote by $A_{i}$, resp. $B_{i}$ any $n \times n$ matrices representing the $a_{i}$, resp. the $b_{i}$ and denote by $X$ the matrix representation of $S$, the conditions for the equality of two quadratic forms given in Section 2.1. allow to immediately translate the quadratic IP1S problem into equivalent matrix equations.

- If the characteristic of $\mathbb{K}$ is odd: the problem is equivalent to finding an invertible matrix $X$ that satisfies the $m$ polar equations: $\mathcal{P}\left(B_{i}\right)={ }^{t} X \mathcal{P}\left(A_{i}\right) X$

- If the characteristic of $\mathbb{K}$ is even: the problem is equivalent to finding an invertible matrix $X$ that satisfies the polar and the diagonal equations: $\mathcal{P}\left(B_{i}\right)={ }^{t} X \mathcal{P}\left(A_{i}\right) X ; \Delta\left(B_{i}\right)=\Delta\left({ }^{t} X A_{i} X\right)$.

In the following sections we will consider IP1S instances such that $m=2$, that are believed to represent the most "interesting" instances of IP1S as reminded above. Matrix pencils, that can be viewed as $n \times n$ matrices whose coefficients are polynomials of degree 1 of $\mathbb{K}[\lambda]$ represent a convenient way to capture the above equations in a more compact way. If we denote by $A$ and $B$ the matrix pencils $\lambda A_{0}+A_{1}$ and $\lambda B_{0}+B_{1}$, and by extension $\mathcal{P}(A)$ and $\mathcal{P}(B)$ the symmetric matrix pencils $\lambda \mathcal{P}\left(A_{0}\right)+\mathcal{P}\left(A_{1}\right)$ and $\lambda \mathcal{P}\left(B_{0}\right)+\mathcal{P}\left(B_{1}\right)$, the two polar equations can be written in one equation: $\mathcal{P}(B)={ }^{t} X \mathcal{P}(A) X$. However, as detailed in the next section, the theory of pencils is far more powerful than just a convenient notation for pairs of matrices. See for instance [3].

\subsection{Mathematical Background}

In this Section we briefly outline a few known definitions and results related to the classification of matrices and matrix pencils and known methods for solving matrix equations that are relevant for the investigation the IP1S problem.

\footnotetext{
${ }^{3}$ While in $[16$ the isomorphism of two $m$-tuples quadratic polynomials comprising also linear and constant terms through a non-singular affine transformation was considered, we consider here the isomorphism of two $m$-tuples of quadratic forms through a non-singular linear transformation. This replacement of the original definition by a simplified definition is justified by the fact that all instances of the initial problem can be shown to be either easily solvable due to the lower degree homogeneous equations they induce or efficiently reducible to an homogeneous quadratic instance.
} 
Basic Facts about Matrices. Two matrices $A$ and $B$ are similar if there exists an invertible matrix $P$ such that $P^{-1} A P=B$ and congruent if there exists an invertible $P$ such that ${ }^{t} P A P=B$.

The matrix $A$ is called cyclic if its minimal and characteristic polynomials are equal.

For any matrix $A$, the commutant of $A$ is the algebra $\mathcal{C}_{A}$ of all matrices commuting with $A$. It contains the algebra $\mathbb{K}[A]$, and this inclusion is an equality if, and only if, $A$ is cyclic.

For any matrix $A$, let $\prod p_{i}^{e_{i}}$ be the prime factorization of its minimal polynomial. Then $\mathbb{K}[A]$ is the direct product of the algebras $\mathbb{K}[x] / p_{i}(x)^{e_{i}}$; each of these factors is a local algebra with residual field equal to the extension field $\mathbb{K}[x] / p_{i}$.

Pencils of Bilinear and Quadratic Forms. Let $V$ be a $\mathbb{K}$-vector space and $Q(V)$ be the vector space of all quadratic forms on $V$. A projective pencil of quadratic forms on $V$ is a projective line in $\mathbb{P} Q(V)$, i.e. a two-dimensional subspace of $Q(V)$. As a projective pencil is the image of the projective line $\mathbb{P}^{1}$ in $Q(V)$, it is determined by the images of the points $\infty$ and 0 in $\mathbb{P}^{1}$, which we write $A_{0}$ and $A_{\infty}$.

An affine pencil of quadratic forms is an affine line in $Q(V)$, or equivalently a pair of elements of $Q(V)$. The affine pencil with basis $\left(A_{\infty}, A_{0}\right)$ may also be written as a polynomial matrix $A_{\lambda}=A_{0}+\lambda A_{\infty}$. Given a projective pencil $A$ of $Q(V)$, the choice of any basis $\left(A_{\infty}, A_{0}\right)$ of $A$ determines an affine pencil.

A projective pencil is regular if it contains at least one regular quadratic form. An affine pencil $\left(A_{\infty}, A_{0}\right)$ is regular if $A_{\infty}$ is regular; it is degenerate if the intersection of the kernels of the quadratic forms $A_{\lambda}$ is nontrivial.

If an affine pencil is non-degenerate, then the polynomial $\operatorname{det} A_{\lambda}$ is non-zero; choosing any $\lambda$ which is not a root of this polynomial proves that the associated projective pencil is regular (over $\mathbb{K}$ itself if it is infinite, and over a finite extension of $\mathbb{K}$ if it is finite). This gives a basis of the projective pencil which turns the affine pencil into a regular one. We shall therefore assume all affine pencils to be regular.

Two pencils $A, B$ of quadratic forms are congruent if there exists an invertible matrix $X$ such that ${ }^{t} X A_{\lambda} X=B_{\lambda}$. The case $m=2$ of the quadratic IP1S problem reduces to the Pencil congruence problem: given two affine pencils $A$ and $B$, known to be congruent, exhibit a suitable congruence matrix $X$.

We first note that the IP1S problem easily reduces to the case where both pencils are regular. Namely, if one (and therefore both) is degenerate, then we may quotient out both spaces by the (isomorphic) kernels of the pencils; this defines non-degenerate affine pencils on the quotient vector spaces, which are still congruent. Since the associated projective pencils are regular, a change of basis in the pencils (and maybe an extension of scalars) brings us to the case of two regular affine pencils.

We define pencils of bilinear forms in the same way as pencils of quadratic forms. The pencil $b_{\lambda}=b_{0}+\lambda b_{\infty}$ regular if $b_{\infty}$ is; in this case, the characteristic endomorphism of the pencil is the endomorphism $f=b_{\infty}^{-1} \circ b_{0}$. 
The following lemma allows to decompose pencils as direct sums, with each factor having a power of an irreducible polynomial as its characteristic endomorphism.

Lemma 1. Let $b$ be a regular pencil of symmetric bilinear forms. Then all primary subspaces of the characteristic endomorphism $f$ are orthogonal with respect to all forms of $b$.

Proof. We have to prove the following: given any two mutually prime factors $p, q$ of $f$ and any $x, y \in V$ such that $p(f)(x)=0$ and $q(f)(y)=0$, then for all $\lambda$, we have $b_{\lambda}(x, y)=0$. For this it is enough to show that $b_{\infty}(x, y)=0$.

Since $p, q$ are mutually prime, there exist $u, v$ such that $u p+v q=1$. Note that, for all $x, y \in V$, we have $b_{\infty}(x, f y)=b_{0}(x, y)=b_{0}(y, x)=b_{\infty}(f x, y)$; therefore, all elements of $\mathbb{K}[f]$ are self-adjoint with respect to $b_{\infty}$. From this we derive the following:

$$
\begin{aligned}
b_{\infty}(x, y) & =b_{\infty}(x, u(f) p(f) y+v(f) q(f) y) \\
& =b_{\infty}(u(f) p(f) x, y)+b_{\infty}(x, v(f) q(f) y) \\
& =0 .
\end{aligned}
$$

Explicit Similarity of a Matrix and Its Transposed. The next result is intensively used in the sequel to deal with symmetric pencils. Although this result is classic [23], we are interested with the explicit form given below.

Theorem 1. For any matrix $M$, there exists a non-singular symmetric matrix T such that ${ }^{t} M T=T M$.

Proof. Using primary decomposition for $M$, we may assume that it is of the form

$$
M=\left(\begin{array}{cccc}
M_{0} & 1 & & 0 \\
& \ddots & \ddots & \\
& & \ddots & 1 \\
0 & & & M_{0}
\end{array}\right),
$$

where $M_{0}$ is the companion matrix of a polynomial $p(\lambda)=\lambda^{n}+\sum_{i=0}^{n-1} p_{i} \lambda^{i}$. We then define matrices $T_{0}$ and $T$ by

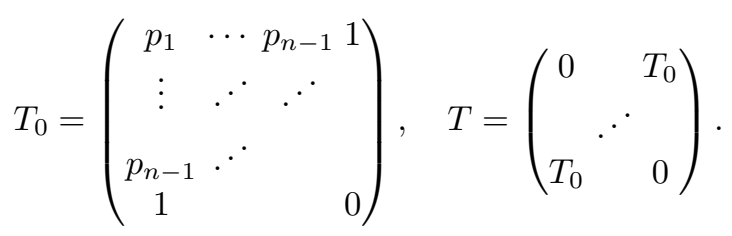

One can easily verify that $T_{0}$ is invertible, symmetric and ${ }^{t} M_{0} T_{0}=T_{0} M_{0}$, and that the same is true for $T$ and $M$. 


\section{IP1S in Characteristic Different from Two}

Let $\mathbb{K}$ be a field of characteristic different from twd. In this case, the polarity identity (1) identifies quadratic forms with symmetric bilinear forms, or again with symmetric matrices with entries in $\mathbb{K}$. We shall therefore write a quadratic pencil $A$ as $A_{\lambda}=A_{0}+\lambda A_{\infty}$, where $A_{0}$ and $A_{\infty}$ are symmetric matrices.

Proposition 1. Let $A_{\lambda}=A_{0}+\lambda A_{\infty}, B_{\lambda}=B_{0}+\lambda B_{\infty}$ be two regular affine pencils.

(i) If $A_{\lambda}$ is congruent to $B_{\lambda}$, then the characteristic matrices

$$
M_{A}=A_{\infty}^{-1} A_{0} \quad \text { and } \quad M_{B}=B_{\infty}^{-1} B_{0}
$$

are similar.

(ii) Assume that $M_{A}$ and $M_{B}$ are similar and choose $P$ such that $P^{-1} M_{A} P=$ $M_{B}$. Then ${ }^{t} P A_{\lambda} P={ }^{t} P A_{\infty} P\left(\lambda+M_{B}\right)$.

(iii) Assume that $A_{\lambda}=A_{\infty}(\lambda+M)$ and $B_{\lambda}=B_{\infty}(\lambda+M)$. Then the solutions of the pencil congruence problem are exactly the invertible $X$ such that

$$
X M=M X \quad \text { and } \quad{ }^{t} X A_{\infty} X=B_{\infty} .
$$

Proof. (i). Since $A_{\lambda}$ is regular, $A_{\infty}$ is invertible and we may write $A_{\lambda}=A_{\infty}(\lambda+$ $\left.A_{\infty}^{-1} A_{0}\right)$; likewise, $B_{\lambda}=B_{\infty}\left(\lambda+B_{\infty}^{-1} B_{0}\right)$. Choose $P$ such that ${ }^{t} P A_{\lambda} P=B_{\lambda}$, then

$$
B_{\infty}\left(\lambda+M_{B}\right)={ }^{t} P A_{\lambda} P={ }^{t} P A_{\infty} P\left(\lambda+P^{-1} M_{A} P\right),
$$

which implies $P^{-1} M_{A} P=M_{B}$ as required. The same computations prove (ii).

The equations (5) follows directly from the equality ${ }^{t} X A_{\infty}(\lambda+M) X=$ ${ }^{t} X A_{\infty} X\left(\lambda+X^{-1} M X\right)$.

We now restrict ourselves to the case where the characteristic endomorphism is cyclic.

Proposition 2. Let $A_{\lambda}=A_{\infty}(\lambda+M)$ and $B_{\lambda}=B_{\infty}(\lambda+M)$ be two regular symmetric pencils such that the matrix $M$ is cyclic, that is, its minimal and characteristic polynomials are equal.

Then the solutions $X$ of the pencil congruence problem are the square roots of $A_{\infty}^{-1} B_{\infty}$ in the algebra $\mathbb{K}[M]$.

Proof. Since $M$ is cyclic, its commutant is reduced to the algebra $\mathbb{K}[M]$; therefore, all solutions of the congruence problem are polynomials in $M$.

Since $A_{\lambda}$ is symmetric, both matrices $A_{\infty}$ and $A_{0}=A_{\infty} M$ are symmetric; therefore, ${ }^{t} M A_{\infty}=A_{\infty} M$. Since $X$ is a polynomial in $M$, we deduce that also ${ }^{t} X A_{\infty}=A_{\infty} X$.

The relation ${ }^{t} X A_{\infty} X=B_{\infty}$ may therefore be rewritten as $A_{\infty} X^{2}=B_{\infty}$, or $X^{2}=A_{\infty}^{-1} B_{\infty}$.

\footnotetext{
${ }^{4}$ Although this is not used in cryptography, we mention that this section also applies
} verbatim to the case of characteristic zero. 
Theorem 2. Let $\mathbb{K}$ be a finite field of odd characteristic and $A_{\lambda}, B_{\lambda}$ be two regular pencils of quadrics over $\mathbb{K}^{n}$, congruent to each other, such that at least one is cyclic (and therefore both are). Then the pencil congruence problem may be solved using no more than $\widetilde{O}\left(n^{3}\right)$ operations in the field $\mathbb{K}$.

Proof. The first step is to reduce to the case of primary components of the characteristic endomorphism. This may be done, using for example Frobenius reduction of both matrices $A_{\infty}^{-1} A_{0}$ and $B_{\infty}^{-1} B_{0}$, with a complexity of $\widetilde{O}\left(n^{3}\right)$ operations. This also provides the change of basis making the characteristic endomorphism of both pencils to have the same matrix.

There remains to compute a square root of $C=A_{\infty}^{-1} B_{\infty}$ in $\mathbb{K}[M]$, where now the minimal polynomial of $M$ is $p^{e}$, with $p$ irreducible. For this we first write $C$ as a polynomial $g(M)$; this again requires $\widetilde{O}\left(n^{3}\right)$ operations. To solve the equation $y^{2}=g(M)$ in the ring $\mathbb{K}[M]=\mathbb{K}[x] / p(x)^{e}$, we first solve it in the (finite) residual field $\mathbb{K}[x] / p(x)$, with complexity $\widetilde{O}\left(n^{3}\right)$ again; lifting the solution to the ring $\mathbb{K}[M]$ requires only $\widetilde{O}\left(n^{2}\right)$ with Hensel lifting.

Solutions of the IP1S problem are square roots of an element $C$ of the algebra $\mathbb{K}[M]$; therefore, the number of solutions is $2^{s}$, where $s$ is the number of connected components of $\mathbb{K}[M]$, that is, the number of prime divisors of the minimal polynomial of $M$.

Summary and Computer Experiments. The case where all the elementary divisors of $\mathcal{P}(A)$ are pairwise co-prime - or equivalently where $M$ is cyclic - represents in practice a quite large fraction of random cases (see for instance [15]). In this case, as shown above, the number of solutions is exactly $2^{s}$ where $s$ is the numbers of elementary divisors and solutions can be efficiently computed (in polynomial time $\widetilde{O}\left(n^{3}\right)$ ) by our method. The highlighted structure of the equations also provides some likely explanations of why Gröbner basis computation methods such as those presented in [2] were successful in this case. We give in next table results (timings) of our MAGMA script SolveCYCLICODdPC, $t$ is the mean execution time when solving 100 random cyclic IP1S instances, $\tau$ is the observed fraction in percent of such "cyclic" instances over random instances.

\begin{tabular}{|c|c|c|c|c|c|c|c|c|c|c|c|}
\hline$q$ & $n$ & $t$ & $\tau$ & $q$ & $n$ & $t$ & $\tau$ & $q$ & $n$ & $t$ & $\tau$ \\
\hline 3 & 80 & 5.s. & 87. & 5 & 20 & $0.07 \mathrm{~s}$. & 95. & $7^{6}$ & 32 & 11.s. & 100. \\
\hline 3 & 128 & 34.s. & 88. & 5 & 32 & $0.28 \mathrm{~s}$ & $\begin{array}{l}95 . \\
05\end{array}$ & \begin{tabular}{|l}
65537 \\
\end{tabular} & 8 & $0.04 \mathrm{~s}$. & 100. \\
\hline $3^{10}$ & 32 & 15.s. & 100. & $\frac{5}{5^{7}}$ & $\frac{80}{32}$ & 8.s. & 100 & 65537 & 20 & 1.s. & 100. \\
\hline
\end{tabular}

\section{IP1S in Characteristic Two}

Let $\mathbb{K}$ be a perfect field of characteristic two. In this case, the polarity identity (1) shows that the polar form $b=\mathcal{P}(q)$ attached to a quadratic form $q$ is an alternating bilinear form. 


\subsection{Pencils of Alternating Bilinear Forms}

This paragraph is a reminder of classical results. We refer the reader to [14 for the proofs.

If $b$ is alternating and nondegenerate, then the vector space $V$ has a symplectic basis, i.e. a basis $\left(e_{1}, \ldots, e_{n}, f_{1}, \ldots, f_{n}\right)$ such that $b\left(e_{i}, f_{i}\right)=1$ and all other pairings are zero. In particular, the dimension of $V$ is even. The vector $E$ space generated by the $e_{i}$ is equal to its orthogonal space $E^{\perp}$; such a space is called a Lagrangian space for $b$.

We recall that two matrices $A$ and $B$ define the same quadratic form if and only if $\mathcal{P}(A)=\mathcal{P}(B)$ and $\Delta(A)=\Delta(B)$.

Although quadratic forms only produce alternating bilinear forms in characteristic two, the following lemma about alternating forms is true in all characteristics. It proves that there exists a basis of $V$ in which the pencil has the block-matrix decomposition

$$
A_{\infty}=\left(\begin{array}{cc}
0 & 1 \\
1 & 0
\end{array}\right), \quad A_{0}=\left(\begin{array}{cc}
0 & { }^{t} F \\
F & 0
\end{array}\right) ; \quad A_{\infty}^{-1} A_{0}=\left(\begin{array}{cc}
F & 0 \\
0 & { }^{t} F
\end{array}\right) .
$$

The matrix $F$ is called the Pfaffian endomorphism of $A$.

Lemma 2. Let $b=\left(b_{\infty}, b_{0}\right)$ be a regular pencil of alternating bilinear forms on $V$. Then there exists a symplectic basis for $b_{\infty}$ whose Lagrangian is stable by the characteristic endomorphism of $b$.

Proof. Let $f$ be the characteristic endomorphism of $b$. By Lemma 1, we may replace $V$ by one of the primary components of $f$ and therefore assume that the minimal polynomial of $f$ is $p^{n}$ where $p$ is a prime polynomial. By extending scalars to $\mathbb{K}[\lambda] / p(\lambda)$ and replacing $b_{0}$ by $\lambda b_{\infty}+b_{0}$ we may assume that $p(t)=t$. We now prove the lemma by induction on $\operatorname{dim} V$.

Since $t^{n}$ is the minimal polynomial of $f$ and $b_{\infty}$ is non-degenerate, there exists $x, y \in V$ such that $b_{\infty}\left(x, f^{n-1} y\right)=1$. Let $W=\mathbb{K}[f] x \oplus \mathbb{K}[f] y$. Then we may write $V=W \oplus W^{\perp}$ where both $W$ and its $b_{\infty}$-orthogonal $W^{\perp}$ are stable by $f$; since $W^{\perp}$ satisfies the lemma by the induction hypothesis, we only need to prove it for $W$.

Let $a(t)=1+a_{1} t+\cdots+a_{n-1} t^{n-1}$ be a polynomial and $x^{\prime}=a(f) x$. Then we still have $b_{\infty}\left(x^{\prime}, f^{n-1} y\right)=1$, and moreover we can choose $a$ so that $b_{\infty}\left(x^{\prime}, f^{i} y\right)=$ 0 for all $i=0, \ldots, n-2$. In other words, $\left(x^{\prime}, f x^{\prime}, \ldots, f^{n-1} x^{\prime}, f^{n-1} y, f^{n-2} y, \ldots\right.$, $f y, y)$ is a symplectic basis for $b_{\infty}$ on $W$. By construction, its Lagrangian is $\mathbb{K}[f] x$, which is obviously stable by the characteristic endomorphism $f$.

Proposition 3. Let $\mathbb{K}$ be a binary field. Any regular pencil of alternating bilinear forms is congruent to a pencil of the form

$$
A_{\infty}=\left(\begin{array}{cc}
0 & T \\
T & 0
\end{array}\right), \quad A_{0}=\left(\begin{array}{cc}
0 & T M \\
T M & 0
\end{array}\right)
$$

where $M$ is in rational (Frobenius) normal form and $T$ is the symmetric matrix defined in Theorem 1 . 
Proof. From the equation [7, choose a matrix $P$ such that $M=P^{-1} F P$ is in rational normal form and define $T$ as in Theorem 1. Then the coordinate change $\left(\begin{array}{ll}P & 0 \\ 0 & { }^{t} P^{-1} T\end{array}\right)$ produces the required form.

Let $A$ be a pencil as in (8). The automorphism group $O(A)$ of $A$ is the set of matrices $X=\left(\begin{array}{ll}X_{1} & X_{2} \\ X_{3} & X_{4}\end{array}\right)$ such that ${ }^{t} X A X=A$, that is all $X_{i}$ commute with $M$ and ${ }^{t} X_{1} T X_{4}+{ }^{t} X_{3} T X_{2}=T$.

From now, we suppose that $M$ is cyclic and for the sake of simplicity that its primary decomposition has only one component.

Since $M$ is cyclic, all $X_{i}$ belong to $\mathbb{K}[M]$. The group $O(A)$ is generated by the elementary transformations

$$
G_{1}(X)=\left(\begin{array}{ll}
1 & X \\
0 & 1
\end{array}\right), \quad G_{2}(X)=\left(\begin{array}{ll}
1 & 0 \\
X & 1
\end{array}\right), \quad G_{3}(X)=\left(\begin{array}{cc}
X & 0 \\
0 & X^{-1}
\end{array}\right), \quad G_{4}=\left(\begin{array}{ll}
0 & 1 \\
1 & 0
\end{array}\right),
$$

where $X \in \mathbb{K}[M], X$ invertible for $G_{3}(X)$. The first three transformations generate the subgroup of positive automorphisms of $A$. This is a subgroup of order two of the orthogonal group [6].

\subsection{Pencils of Quadratic Forms}

The following proposition deals with the diagonal terms of a quadratic form in the cyclic case. We recall that, using the notations of Theorem $\mathbb{1}, \mathbb{K}\left[M_{0}\right]$ is an extension field of $\mathbb{K}$, and $\mathbb{K}[M]$ is the (local) $\mathbb{K}\left[M_{0}\right]$-algebra generated by

$$
H=\left(\begin{array}{cccc}
0 & 1 & & 0 \\
& \ddots & \ddots & \\
& & \ddots & 1 \\
0 & & & 0
\end{array}\right)
$$

We write $\varphi(X)=X^{2}$ for the Frobenius map of $\mathbb{K}\left[M_{0}\right]$. Since this is a finite field, the Frobenius map is bijective. It extends to $\mathbb{K}[M]$ as $\varphi\left(\sum x_{i} H^{i}\right)=\sum x_{i}^{2} H^{i}$.

Proposition 4. Define matrices $M$ of size $n, M_{0}, T_{0}$ of size $e=n / d$ as in Theorem 1 .

(i) The $\mathbb{K}$-linear map $\mathbb{K}\left[M_{0}\right] \mapsto \mathbb{K}^{e}, X \mapsto \Delta\left(T_{0} X\right)$ is an isomorphism.

(ii) For any diagonal matrix $D$ of size $e$, there exists a (unique) matrix $C=$ $\psi_{0}(D) \in \mathbb{K}\left[M_{0}\right]$ such that, for all $X \in \mathbb{K}\left[M_{0}\right]$ :

$$
\Delta\left({ }^{t} X D X\right)=\Delta\left(T_{0} C X^{2}\right) .
$$

(iii) Let $D$ be a diagonal matrix of size $n$, written as blocks $D_{0}, \ldots, D_{d-1}$, and write $X \in \mathbb{K}[M]$ as $X=\sum x_{i} H^{i}$ with $x_{i} \in \mathbb{K}\left[M_{0}\right]$. Also define $\psi(D)=$ $\sum \psi_{0}\left(D_{i}\right) H^{i} \in \mathbb{K}[M]$. Then we have the relation in $\mathbb{K}[M]$

$$
\psi\left(\Delta\left({ }^{t} X D X\right)\right)=\varphi(X) \cdot \psi(D) .
$$


Proof. (i) Since $2=0$ in $\mathbb{K}$, for any symmetric matrix $A$ and any $X$, we have

$$
\Delta\left({ }^{t} X \Delta(A) X\right)=\Delta\left({ }^{t} X A X\right) .
$$

Since the space $\mathbb{K}\left[M_{0}\right]$ has dimension $e$ over $\mathbb{K}$, we only have to check injectivity. Assume $\Delta\left(T_{0} X\right)=0$ with $X \neq 0$; since $\mathbb{K}\left[M_{0}\right]$ is a field, $X$ is invertible. Let $Y=$ $\varphi^{-1}\left(X^{-1}\right)$. We then have

$$
\Delta\left(T_{0}\right)=\Delta\left(T_{0} X Y^{2}\right)=\Delta\left({ }^{t} Y\left(T_{0} X\right) Y\right)=\Delta\left({ }^{t} Y \Delta\left(T_{0} X\right) Y\right)=0 .
$$

Let $p(x)=p_{0}+\cdots+p_{e-1} x^{e-1}+x^{e}$ be the minimal polynomial of $M_{0}$. From $\Delta\left(T_{0}\right)$ $=0$ we deduce that $p_{e-1}=p_{e-3}=\cdots=0$, which contradicts the irreducibility of $p$.

(ii) Let $C \in \mathbb{K}\left[M_{0}\right]$ such that $\Delta(C)=D$; applying (13) to the symmetric matrix $T_{0} C$ and using the symmetry of $T_{0} M_{0}$ yields

$$
\Delta\left(T_{0} C X^{2}\right)=\Delta\left({ }^{t} X T_{0} C X\right)=\Delta\left({ }^{t} X \Delta\left(T_{0} C\right) X\right)=\Delta\left({ }^{t} X D X\right) .
$$

(iii) From direct computation we find that the diagonal blocks of ${ }^{t} X D X$ are $B_{m}=\sum_{i+j=m}{ }^{t} X_{i} D_{j} X_{i}$; hence $\Delta\left(B_{m}\right)=\sum \Delta\left(T_{0} \psi_{0}\left(D_{j}\right) X_{i}^{2}\right)$ and $\psi_{0}\left(B_{m}\right)=$ $\sum \psi_{0}\left(D_{j}\right) \varphi\left(X_{i}\right)$.

For any binary field $\mathbb{K}$, we write $\wp(\mathbb{K})$ for the set of elements $x^{2}+x \in \mathbb{K}$. This is an additive subgroup of $\mathbb{K}$, and the characteristic-two analogue of the set of squares. For any element $\alpha$ of $\mathbb{K}[M]$, we call valuation of regularity of $\alpha$ that we simply note $\operatorname{val}(\alpha)$ the smallest integer $m$ such that there exists an invertible $\alpha^{\prime}$ of $\mathbb{K}[M]$ such that $\alpha=H^{m} \alpha^{\prime}$.

Proposition 5. Any regular pencil of quadratic forms is congruent to a pencil of the form

$$
A_{\infty}=\left(\begin{array}{cc}
D_{1} & T \\
0 & D_{2}
\end{array}\right), \quad A_{0}=\left(\begin{array}{cc}
D_{3} & T M \\
0 & D_{4}
\end{array}\right)
$$

where $M, T$ are as in Prop. 3 and $D_{i}$ are diagonal matrices whose values $\alpha_{i}=$ $\psi\left(D_{i}\right)$ satisfy either one or the other of the following two kinds of canonical forms:

(i) $\alpha_{1}=H^{m}, \operatorname{val}\left(\alpha_{1}+\alpha_{3}\right)>m, \alpha_{2}=0$ or $\alpha_{2}=\delta H^{d-1-m}, \operatorname{val}\left(\alpha_{4}\right) \geq m$, for some $m \in\{0, \ldots, d\}$, and some fixed $\delta \in \mathbb{K}\left[M_{0}\right] \backslash \wp\left(\mathbb{K}\left[M_{0}\right]\right)$;

(ii) $\alpha_{1}=H^{m}$ or $\alpha_{3}=H^{m}, \operatorname{val}\left(\alpha_{1}+\alpha_{3}\right)=m, \alpha_{2}=\alpha_{4}, \operatorname{val}\left(\alpha_{2}\right)>m$ for some $m \in\{0, \ldots, d\}$, and some fixed $\delta \in \mathbb{K}\left[M_{0}\right] \backslash \wp\left(\mathbb{K}\left[M_{0}\right]\right)$.

Proof. By Prop. 3, we may compute bases in which the pencils of polar forms have the form (8). In the same bases the pencils have the form (16) with $M, T$, $M_{0}, T_{0}$ as in Theorem 1 and $D_{i}$ are some diagonal matrices. We now perform elementary transformations of the orthogonal group of $\mathcal{P}(A)$ to simplify the diagonal part of the quadratic pencil. We use the transformations $G_{i}(X)$ from (9) 
for a matrix $X=x_{0}+\cdots+x_{d-1} H^{d-1} \in \mathbb{K}[M]$. The effects of the elementary transformations $G_{i}(X)$ on the coefficients $\alpha_{i}$ are:

$$
\begin{aligned}
G_{1}(X): \quad & \alpha_{1} \leftarrow \alpha_{1}+\varphi(X) \alpha_{2}+\psi(\Delta(T X)), \\
& \alpha_{3} \leftarrow \alpha_{3}+\varphi(X) \alpha_{4}+\psi(\Delta(T X)), \\
& \alpha_{2} \leftarrow \alpha_{2}, \quad \alpha_{4} \leftarrow \alpha_{4} ; \\
G_{2}(X): \quad & \alpha_{2} \leftarrow \alpha_{2}+\varphi(X) \alpha_{1}+\psi(\Delta(T X)), \\
& \alpha_{4} \leftarrow \alpha_{4}+\varphi(X) \alpha_{3}+\psi(\Delta(T X)), \\
& \alpha_{1} \leftarrow \alpha_{1}, \quad \alpha_{3} \leftarrow \alpha_{3} ; \\
G_{3}(X): \quad & \alpha_{1} \leftarrow \varphi(X) \alpha_{1}, \quad \alpha_{2} \leftarrow \varphi\left(X^{-1}\right) \alpha_{2}, \\
& \alpha_{3} \leftarrow \varphi(X) \alpha_{3}, \quad \alpha_{4} \leftarrow \varphi\left(X^{-1}\right) \alpha_{4} ; \\
G_{4}: & \alpha_{1} \leftarrow \alpha_{2}, \quad \alpha_{3} \leftrightarrow \alpha_{4} .
\end{aligned}
$$

A direct computation gives

$$
\psi(\Delta(T M X))=\sum_{i \geq \frac{d-1}{2}} x_{2 i-(d-1)} H^{i} .
$$

As in Prop. 4, we write $D_{i}$ as $d$ blocks $D_{i, j}$ and define $\alpha_{i, j}=\psi_{0} D_{i, j}$. From what we get above we explicit the effects of the elementary transformation $G_{1}(X)$ on the coefficients $\alpha_{i, j}$ :

$$
\begin{aligned}
G_{1}(X): \alpha_{1, m} & \leftarrow \alpha_{1, m}+\sum_{i+j=m} \alpha_{2, i} x_{j}^{2} \quad \text { for } m<\frac{d-1}{2} \\
\alpha_{1, m} & \leftarrow \alpha_{1, m}+\sum_{i+j=m} \alpha_{2, i} x_{j}^{2}+x_{2 m-(d-1)} \quad \text { for } m \geq \frac{d-1}{2} ;
\end{aligned}
$$

If all $\alpha_{i}=0$, we are done: the pencil is canonical. If not, we search the value $\alpha_{i}$ with smallest valuation. Using $G_{4}$, we may assume it is $\alpha_{1}$ or $\alpha_{3}$. We first suppose that we have $\operatorname{val}\left(\alpha_{1}+\alpha_{3}\right)>m$, that is $\alpha_{1}$ and $\alpha_{3}$ have the same trailing term. We call this the case (i). Using $G_{3}$, we may assume $\alpha_{1}=H^{m}$, and therefore $\alpha_{3}=H^{m}+\alpha$, with val $(\alpha)>m$. We look then for $X$ such that $G_{2}(X)\left(\alpha_{2}\right)=0$. We note that the corresponding system is triangular and all equations can be solved except maybe for this one: $\alpha_{2, d-1-m}=x_{d-1-2 m}^{2}+x_{d-1-2 m}$. Therefore we may assume that $\alpha_{2}=0$ or $\alpha_{2}=\delta H^{d-1-m}$ for some fixed $\delta \in \mathbb{K}\left[M_{0}\right] \backslash \wp\left(\mathbb{K}\left[M_{0}\right]\right)$. We note also that $G_{2}(X)$ does not decrease the valuation of $\alpha_{4}$. We have therefore by hypothesis $\operatorname{val}\left(\alpha_{4}\right) \geq m$.

We now examine the case (ii) where val $\left(\alpha_{1}+\alpha_{3}\right)=m$. Using again $G_{3}$, we may assume that $\alpha_{1}=H^{m}$ or $\alpha_{3}=H^{m}$. Let's note $\alpha_{1}+\alpha_{3}=H^{m} \alpha$ where $\alpha$ is invertible. We are looking for $X$ such that $G_{2}(X)\left(\alpha_{2}\right)=G_{2}(X)\left(\alpha_{4}\right)$. By hypothesis on the valuation, we can write $\alpha_{2}+\alpha_{4}=H^{m} \alpha^{\prime}$ for some $\alpha^{\prime}$. We naturally choose $X=\varphi^{-1}\left(\alpha^{\prime} \alpha^{-1}\right)$. At this stage, we can consider that $\alpha_{2}=\alpha_{4}$. 
However, the condition on the valuation may not hold. If by chance val $\left(\alpha_{2}\right)>m$, then we are done. If on the contrary $\operatorname{val}\left(\alpha_{2}\right) \leq m$, then by using $G_{4}$, we search instead for a canonical form of the kind (i).

Theorem 3. Let $\mathbb{K}$ be a finite field with characteristic two. The cyclic case of the IP1S problem is solvable using $\widetilde{O}\left(n^{3}\right)$ operations in the field $\mathbb{K}$. Moreover, in the generic case, the IP1S problem has exactly $2^{s}$ solutions, where $s$ is the number of components within the primary decomposition of $M$.

Proof. To solve the IP1S problem for two pencils $A$ and $B$, we may reduce them to the same canonical form using Prop. 5 using first the primary decomposition. Following along the proof of the proposition, we see that it is constructive and that all linear algebra algorithms used require at most $\widetilde{O}\left(n^{3}\right)$ field operations.

Solutions of the IP1S problem correspond bijectively to automorphisms of the canonical pencil. In the generic case, the ideal generated by the values $\left(\alpha_{1}, \alpha_{2}\right)$ is the full algebra $\mathbb{K}[M]$; the canonical pencil is then such that that $\alpha_{1}=1$ and $\alpha_{2} \in\left\{0, \delta H^{d-1}\right\}$.

For both values of $\alpha_{2}$, since the equation $x_{d-1}^{2}+x_{d-1}=0$ has only the solutions 0 and 1 in each component $\mathbb{K}\left[M_{0}\right]$, the IP1S problem has in this case exactly $2^{s}$ solutions.

IP1S Problem for $a$ and $b$ : Summary and Computer Experiments. Next table gives timings of our MAGMA script SolveCYCLICEVENIP1S, with the same convention as for the odd case : $\tau$ represents the observed fraction of cyclic cases and $t$ the average computing time over these cases.

\begin{tabular}{||c|c|r|r||||c|r|r|r||}
\hline$q$ & $n$ & $t$ & $\tau$ \\
\hline \hline 2 & 32 & 0.07 s. & 96. \\
2 & 128 & $2 . s$. & 95. \\
2 & 256 & $33 . s$. & 94. \\
\hline $2^{4}$ & 32 & 0.3 s. & 100. \\
\hline $2^{7}$ & 32 & 0.5 s. & 100. \\
\hline $2^{8}$ & 20 & $0.2 s$. & 100. \\
$2^{8}$ & 32 & $0.6 s$. & 100. \\
$2^{8}$ & 80 & $20 . s$. & 100. \\
$2^{8}$ & 128 & $133 . s$ & 100. \\
\hline
\end{tabular}

\section{Conclusion and Future Work}

We have shown that special instances of the quadratic homogeneous IP1S problem with $m=2$ equations can be solved in polynomial time. These instances are those where the characteristic endomorphism of the pencil (or its Pfaffian when the characteristic of the field is 2) is cyclic, and represent in practice a large fraction of generic instances. In a subsequent work, we studied the case where the characteristic endomorphism is no longer cyclic and found similar results to be published - at least for odd characteristic fields. In a work still in progress, we try to extend these results to QIP1S problem with more than 2 equations, and therefore expect to confirm that QIP1S is not as hard as GI. 


\section{References}

1. Bouillaguet, C.: Études d'hypothèses algorithmiques et attaques de primitives cryptographiques. PhD thesis, Université Paris-Diderot - École Normale Supérieure (2011)

2. Bouillaguet, C., Faugère, J.-C., Fouque, P.-A., Perret, L.: Practical cryptanalysis of the identification scheme based on the isomorphism of polynomial with one secret problem. In: Catalano, et al. [5], pp. 473-493

3. Bouillaguet, C., Fouque, P.-A., Macario-Rat, G.: Practical key-recovery for all possible parameters of sflash. In: Lee, D.H., Wang, X. (eds.) ASIACRYPT 2011. LNCS, vol. 7073, pp. 667-685. Springer, Heidelberg (2011)

4. Bouillaguet, C., Fouque, P.-A., Véber, A.: Graph-theoretic algorithms for the "isomorphism of polynomials" problem. IACR Cryptology ePrint Archive 2012, 607 (2012)

5. Catalano, D., Fazio, N., Gennaro, R., Nicolosi, A. (eds.): PKC 2011. LNCS, vol. 6571. Springer, Heidelberg (2011)

6. Dieudonné, J.: Pseudo-discriminant and Dickson invariant. Pacific J. Math 5, 907$910(1955)$

7. Dubois, V., Kammerer, J.-G.: Cryptanalysis of cryptosystems based on noncommutative skew polynomials. In: Catalano, et al. [5], pp. 459-472

8. Faugère, J.-C.: A new efficient algorithm for computing Gröbner bases (F4). Journal of Pure and Applied Algebra 139(1-3), 61-88 (1999)

9. Faugère, J.-C., Gianni, P., Lazard, D., Mora, T.: Efficient Computation of Zerodimensional Gröbner Bases by Change of Ordering. Journal of Symbolic Computation 16(4), 329-344 (1993)

10. Garey, M.R., Johnson, D.S.: Computers and Intractability: A Guide to the Theory of NP-Completeness. W. H. Freeman \& Co. (1979); Ch. 7.2: Algebraic Equations over $\mathrm{GF}(2)$

11. Goldreich, O.: The Foundations of Cryptography — Volume 1, Basic Techniques. Cambridge University Press (2001)

12. Kipnis, A., Patarin, J., Goubin, L.: Unbalanced oil and vinegar signature schemes. In: Stern, J. (ed.) EUROCRYPT 1999. LNCS, vol. 1592, pp. 206-222. Springer, Heidelberg (1999)

13. Matsumoto, T., Imai, H.: Public quadratic polynomial-tuples for efficient signatureverification and message-encryption. In: Günther, C.G. (ed.) EUROCRYPT 1988. LNCS, vol. 330, pp. 419-453. Springer, Heidelberg (1988)

14. Milnor, J.W., Husemoller, D.: Symmetric bilinear forms. Springer-Verlag (1973)

15. Neumann, P.M., Praeger, C.E.: Cyclic matrices over finite fields. J. London Math. Soc. (2) 52(2), 263-284 (1995)

16. Patarin, J.: Hidden Fields Equations (HFE) and Isomorphisms of Polynomials (IP): Two New Families of Asymmetric Algorithms. In: Maurer, U.M. (ed.) EUROCRYPT 1996. LNCS, vol. 1070, pp. 33-48. Springer, Heidelberg (1996)

17. Patarin, J., Goubin, L., Courtois, N.T.: Improved algorithms for isomorphisms of polynomials. In: Nyberg, K. (ed.) EUROCRYPT 1998. LNCS, vol. 1403, pp. 184-200. Springer, Heidelberg (1998)

18. Patarin, J., Goubin, L., Courtois, N.T.: $C_{-+}^{*}$ and HM: Variations Around Two Schemes of T. Matsumoto and H. Imai. In: Ohta, K., Pei, D. (eds.) ASIACRYPT 1998. LNCS, vol. 1514, pp. 35-50. Springer, Heidelberg (1998)

19. Perret, L.: A fast cryptanalysis of the isomorphism of polynomials with one secret problem. In: Cramer, R. (ed.) EUROCRYPT 2005. LNCS, vol. 3494, pp. 354-370. Springer, Heidelberg (2005) 
20. Pointcheval, D.: A new identification scheme based on the perceptrons problem. In: Guillou, L.C., Quisquater, J.-J. (eds.) EUROCRYPT 1995. LNCS, vol. 921, pp. 319-328. Springer, Heidelberg (1995)

21. Shamir, A.: An efficient identification scheme based on permuted kernels. In: Brassard, G. (ed.) CRYPTO 1989. LNCS, vol. 435, pp. 606-609. Springer, Heidelberg (1990)

22. Stern, J.: Designing identification schemes with keys of short size. In: Desmedt, Y.G. (ed.) CRYPTO 1994. LNCS, vol. 839, pp. 164-173. Springer, Heidelberg (1994)

23. Taussky, O., Zassenhaus, H.: On the similarity transformation between a matrix and its transpose. Pacific J. Math. 9, 893-896 (1959)

\section{A Complexity, Timings, and Other Considerations}

All the experimental results have been obtained with an Opteron $8502.2 \mathrm{GHz}$, with 32 GBytes of Ram. The systems associated with the instance of the problems and their solutions have been generated using the MAGMA software, version 2.13-15. MAGMA scripts cited in this paper can be obtained from the authors. 